\title{
Analog Radio-over-Fiber Fronthaul by a WDM-PON employing Double RSOA Self- Seeding and Carrier-Reuse Techniques
}

\author{
Adelcio M. Souza $^{1}$, Daniel R. Celino ${ }^{1}$, Ulysses R. Duarte ${ }^{2}$, Murilo A. Romero ${ }^{1}$ \\ ${ }^{l}$ Sao Carlos School of Engineering - University of Sao Paulo (EESC/USP), Sao Carlos-SP, Brazil. \\ e-mails: adelcio.souza@usp.br,daniel.celino@usp.br,murilo.romero@usp.br \\ ${ }^{2}$ Federal Institute of Minas Gerais (IFMG), Formiga-MG, Brazil.e-mail: ulysses.rondina@ifmg.edu.br
}

\begin{abstract}
WDM-PON has been considered a promising solution for future fronthaul links concerning new mobile networks applications. However, in order to avoid inventory problems and reduce operation costs, there is a need for colorless sources, where the same basic optical source can be used regardless of the desired wavelength. Also, the digital RoF scheme, which has been employed so far, might require a bandwidth that is prohibitive even for optical fiber links. In this paper, we propose and numerically investigate a bidirectional colorless WDM-PON fronthaul transporting analog RoF signals as an alternative to meet these demands. All simulations were performed using a framework calibrated with experimental data. For the downstream, we employ a double-cavity self-seeding technique, while the upstream is performed by a cascaded-RSOAs carrier-reuse approach. BER and EVM simulation analysis are presented for various data rates, modulations formats and RF bands ranging from 1 to $5 \mathrm{GHz}$, demonstrating the feasibility of our proposed topology as a novel fronthaul approach.
\end{abstract}

Index Terms - Analog Radio-over-Fiber, Carrier Reuse, Mobile Fronthaul, Self-Seeding, WDM-PON.

\section{INTRODUCTION}

Wavelength-Division Multiplexing Passive Optical Networks (WDM-PONs) have been intensively investigated as a solution for the increasing data demand expected in the next years [1]. Unlike traditional Time-Division Multiplexing PON (TDM-PON), a unique wavelength is assigned to each user and there is no resource sharing, providing a higher individual bandwidth. However, one of the main issues, being studied since the early days of WDM-PON research, is the need for different transmitters for each wavelength, which increases inventory and operational costs. Although tunable lasers could be a potential solution for this problem, they are still too expensive for a commercial PON application, albeit some progress recently made [2].

In order to attain a cost-effective solution to this problem, several colorless sources relying on selfseeding technique have been proposed [3]-[7]. In the self-seeding technique, the amplified spontaneous emission (ASE) spectrum of an active device, such as the Reflected Semiconductor Optical Amplifier (RSOA), is sliced accordingly to the assigned wavelength by an Arrayed Wavelength Grating (AWG) and an external cavity self-tunable transmitter is established in between Brazilian Microwave and Optoelectronics Society-SBMO received 3 Oct 2018; for review 5 Nov 2018; accepted 16 Nov 2018 Brazilian Society of Electromagnetism-SBMag 
the rear facet of the RSOA and an additional mirror placed in the optical path. In most cases, these self-seeding schemes were realized at the Optical Network Termination (ONT), yielding a cavity as long as the drop-fiber length (a few kilometers), thereby degrading the quality of the produced carrier. Also, they usually employ two distinct wavelengths for downstream and upstream data flows, limiting the number of channels made available by the network.

Hence, in a previous experimental investigation, we have explored the advantages of generating the self-seeding downstream carrier at the Optical Line Termination (OLT) and employing wavelengthreuse for the upstream [8]. In other words, the downlink data is erased at the ONT and the same carrier generated by self-seeding at the OLT is used for upstream transmission. More recently, aiming to reinforce data erasure and improve performance, we numerically investigated a double-cavity selfseeding scheme with orthogonal polarizations and a cascaded-RSOAs carrier-reuse approach, adding an extra RSOA on both ends [9].

Currently, the main driving application for future PONs is the data transport for mobile networks, given that home and business users are expected to be well served by current Gigabit PON (GPON) standards, at least during the next years [10]. Regarding the mobile network structure, optical fibers are currently widely used for connections to the transport network, the so-called backhaul. In the last decade, the proposition of a Centralized Radio Access Network (C-RAN), where most base-band processing is moved from the Base Station (BS) to the Central Office (CO), has also explored the use of optical fibers [11]. The connection between these entities is known as fronthaul.

Two main protocols have been adopted for mobile fronthaul, Common Public Radio Interface (CPRI) [12] and OBSAI Open Base Station Architecture Initiative (OBSAI) [13]. They are based on Digital Radio-over-Fiber (D-RoF) techniques, where the RF signals are digitalized into IQ samples. Nonetheless, D-RoF might not be suitable for future high speed mobile networks, since it would require an enormous bandwidth. This is why Analog Radio-over-Fiber (A-RoF) has been appointed as a feasible and less expensive fronthaul alternative [14]. In A-RoF, mobile signals are sent in their original waveforms by a RF carrier over the fiber, in such way that the required bandwidth can be drastically reduced.

During the last years, there were successful efforts to combine self-seeding techniques and RoF transport for mobile applications [15]-[17]. Yet, all of them are based on the CPRI protocol and the self-seeding process is carried out on the ONT side. In this paper, we propose the transport of A-RoF using a bidirectional WDM-PON which employs double-cavity self-seeding on the OLT side and cascaded-RSOAs carrier-reuse on the ONT side as an alternative for mobile fronthaul. To the best of our knowledge, this is the first investigation regarding the transport of analog RF signals in a WDMPON, in general, and certainly true in a specific configuration relying on self-seeding and carrierreuse techniques. A preliminary version of the work, comprising only the downlink analysis was presented at the conference MOMAG, hosted by the Instituto Nacional de Telecomunicações at Santa Rita do Sapucaí - MG in 2018 [18]. In the present work we also included an analysis for 16QAM Brazilian Microwave and Optoelectronics Society-SBMO received 3 Oct 2018; for review 5 Nov 2018 ; accepted 16 Nov 2018 Brazilian Society of Electromagnetism-SBMag 2018 SBMO/SBMag $\quad$ (cc)) BY 
downstream transmission. In addition, we investigated the transmission in the upstream direction, erasing the downstream data and reusing the carrier to transmit back the same signal format.

The proposed bidirectional topology will be described on Section II. In order to evaluate the technical feasibility of the proposal, we first calibrated a simulation framework with our experimental data [8], as it will be described on Section III, and then studied the proposed topology using Optisystem 13, as detailed in Section IV. The results and discussion on the transmission of various data rates, modulations formats and RF bands ranging from 1 to $5 \mathrm{GHz}$ on both directions are presented on Section V. Finally, on Section VI, we draw the conclusions about this work.

\section{ANALOG FRONTHAUL TOPOLOGY BASED ON SELF-SEEDING COLORLESS SOURCES}

The traditional Base Station is usually composed of two main elements: the Base Band Unit (BBU), where all digital processing takes place, and the Radio Unit (RU), responsible for all RF processing. In the case of C-RAN, the BBUs are located in the Central Office or even virtualized in the cloud, leaving the BS as a simple Remote Radio Head (RRH). As a result of the simplified radio unit, the cell site consumes less energy, it is easier to install and maintain and the need for air-cooling can be eliminated. On the other hand, the BBUs are able to communicate among themselves with lower delays and cooperate to perform complex tasks, such as flexible response to non-uniform traffic and Coordinate Multi-Point (CoMP). Also, heterogeneous networks and services can largely benefit from resource and information sharing.

Although CPRI have been largely implemented for Long Term Evolution (LTE) and even earlier mobile networks standards, the next generations might not benefit from this D-RoF solution. This is because, to fulfill the upcoming data demands, future mobile coverage is expected to be implemented using numerous high-speed small cells instead of isolated macro-cells. As the number of cell increases, more fronthaul links will be necessary, each one with increasingly prohibitive bandwidth consumption. For instance, today, a simple LTE scenario with a single sector, 2x2 Multiple Input Multiple Output (MIMO) and a $20 \mathrm{MHz}$ channel would require a 2.458 Gbps fronthaul link [11]. A LTE Advanced (LTE-A) scenario supporting downlink peak data rates of 1 Gbps would require a 147.456 Gbps fronthaul link [19]. In contrast, an estimation of a potential next mobile generation $(5 \mathrm{G})$ indicated it might need a prohibitive 2.359 Tbps fronthaul link with current CPRI [20]. Additionally, the digitalization process introduces latencies and jitter, which will have very strict constraints in the upcoming mobile networks.

Various techniques to reduce the sample rate, such as non-linear quantization and IQ data compression have been proposed [21]-[23], but there is a trade-off between the compression ratio and signal distortion, limiting their efficiency. These techniques also come with the drawback of additional complexity and computational power. Therefore, it has been suggested that even further centralization will be needed, with Analog RoF being a promising solution. The required optical data rate can be reduced to the effective mobile data rate and, since all digital processing can be eliminated 
from the Radio Unit, the only fronthaul delay mechanism is the propagation time relative to the link length. Moreover, some of the RF processing can be performed in the $\mathrm{CO}$ or even along the link, like all optical RF amplification [21].

In terms of costs, a comparative study between CPRI, A-RoF and a functional split solution called Physical Layer Split (PLS) was recently published by Ranaweera et al. [24]. They demonstrated that PLS and ARoF both satisfy the primary requirements of $5 \mathrm{G}$ and the deployment costs are reduced when compared to the traditional CPRI-based C-RAN. On the other hand, the main drawback of analog transmission over a fiber link is the higher susceptibility to chromatic dispersion and nonlinearities. Regarding chromatic dispersion, the main constraint is the fading effect, where the beating of side-bands leads to periodic destructive interference [25]. However, this problem has been addressed with chromatic dispersion compensation, zero dispersion fibers and special optical modulation formats [26]. The recent interest on A-RoF for mobile fronthaul has also boosted the investigation on various nonlinearities mitigation techniques [27], [28].

Another critical requirement for the future fronthaul is to be able to serve multiple small-cells with several antennas each, delivering high speed data. One of the best alternatives is to employ a colorless WDM-PON structure, where several dedicated links can be established and the inventory problem is mitigated. WDM-PON was already considered during the development of Next Generation PON 2 (NGPON2) standard [1] and it is a promising option for future standardization.

In this context, we propose here a novel fronthaul topology, as illustrated in Fig. 1. It comprises a C-RAN architecture where the RRH becomes a Radio Access Unit (RAU), composed only by E/O and $\mathrm{O} / \mathrm{E}$ converters and basic RF processing, such as amplification, filtering, etc. The optical links serving this fronthaul architecture are part of a WDM-PON, which employs a double-cavity selfseeding transmitter for downlink data flow and a cascaded-RSOAs carrier-reuse transmitter for the uplink direction.

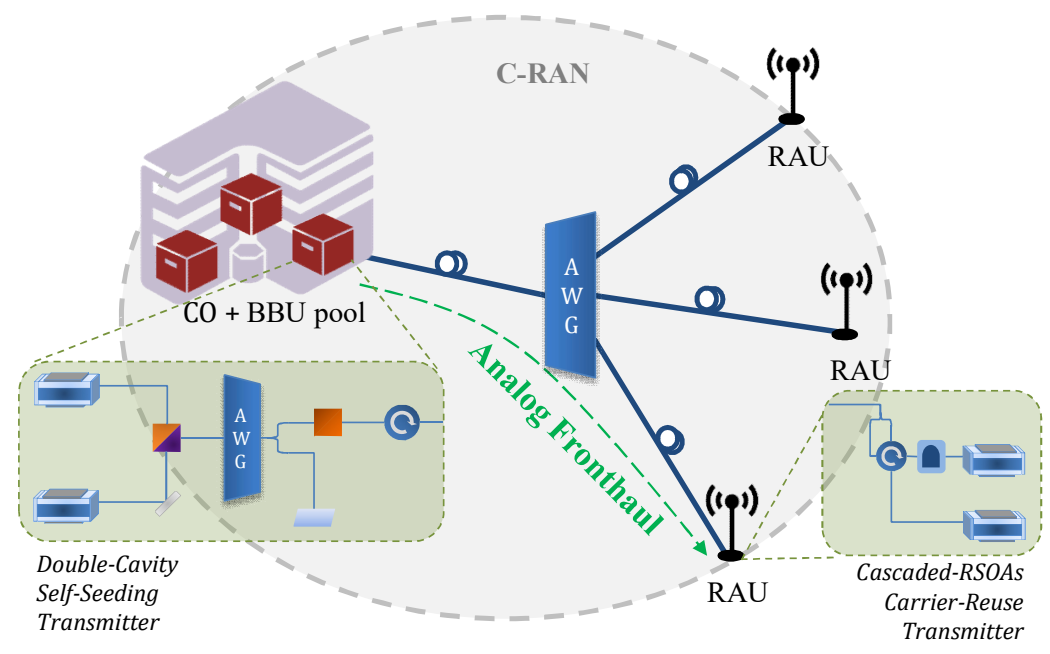

Fig. 1. Proposed analog fronthaul over a WDM-PON, combining a double-cavity self-seeding technique (downlink) and a cascaded-RSOAs carrier-reuse technique (uplink). Apart from minute differences on operating wavelength, all logical links are essentially identical and, therefore, a single optical link will be considered for simulation. 
In our earlier work, we experimentally demonstrated a combined self-seeding and carrier-reuse WDM-PON topology. A detailed description of this topology can be found in [8]. The RSOAs available for the experiment presented low gain profile, with about $21 \mathrm{~dB}$ of maximum gain and a saturation power of $5 \mathrm{dBm}$. Consequently, the circulating optical power was not enough to achieve self-stability in the established self-seeding cavity. As a first approach, an Erbium-Doped Fiber Amplifier (EDFA) and a set of optical circulators were coupled to the RSOA to overcome the cavity losses, reaching an effective lasing regime. The results demonstrated an error free downstream transmission for extinction ratios as low as $3.5 \mathrm{~dB}$.

In order to enhance the erasure efficiency and, in addition, eliminate the need for an expensive EDFA, we later proposed a double-cavity self-seeding topology [29] (Fig. 2). This topology uses two RSOAs on the OLT: the first one, called RSOA T $x_{\mathrm{D}}$, is directly modulated with downstream data while the other one, called RSOA Aux $x_{D}$, is DC biased to provide the extra erasure. Also, a set-up comprising a Polarization Beam Splitter (PBS), a Half-Wave Plate (HWP) and Faraday Rotator Mirror (FRM) ensures the maintenance of the state of polarization of the two orthogonal polarizations in the recirculation path, as described in [7].

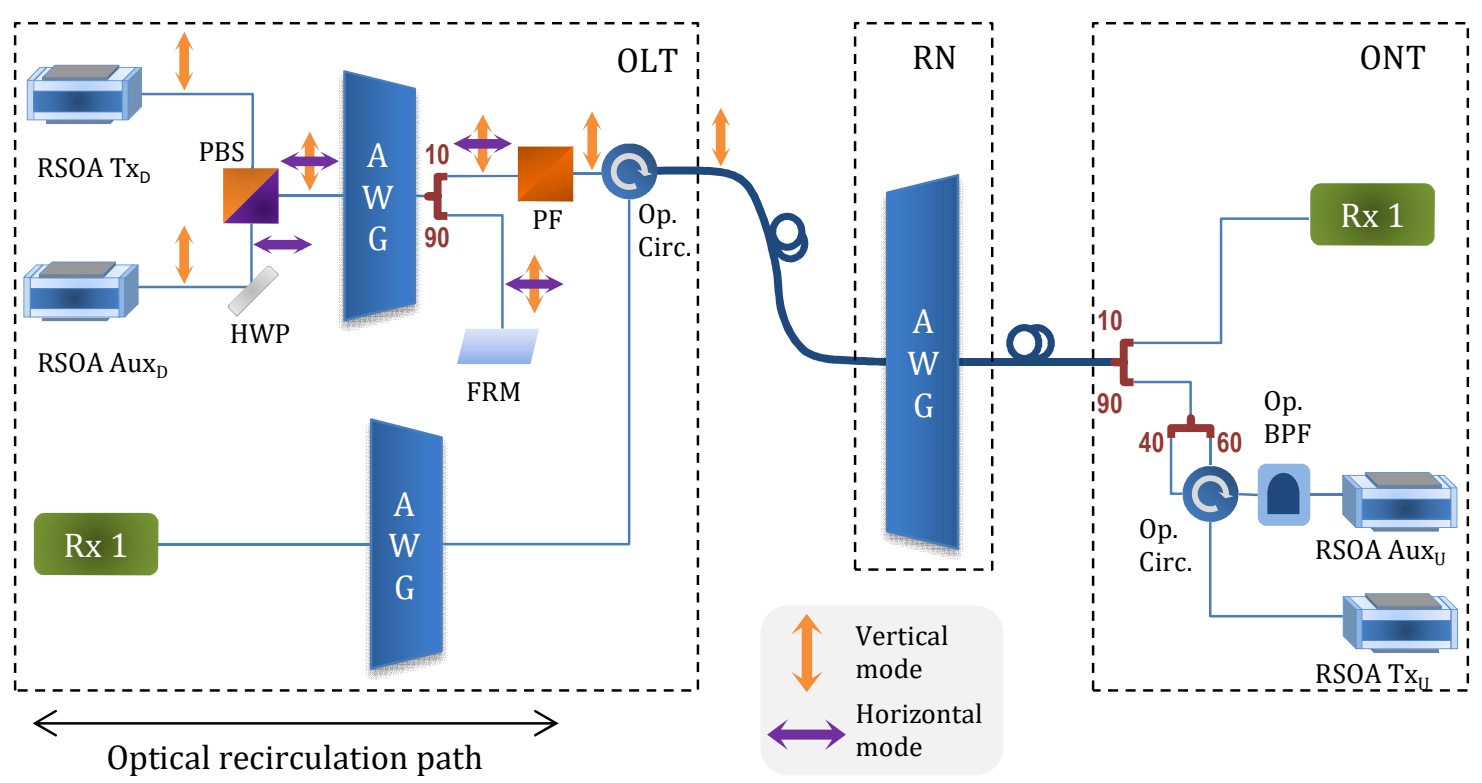

Fig. 2. Combined double-cavity self-seeding and cascaded-RSOAs carrier-reuse WDM-PON link topology. The two orthogonal cavities formed at the OLT are highlighted with their states of polarization.

In the first-round trip, the optical signal generated by RSOA $\mathrm{Tx}_{\mathrm{D}}$ goes through the PBS and emerges polarized in the Vertical Mode (VM). An AWG selects the designated wavelength and 90\% of the available optical power is directed to the FRM by the optical coupler. At the FRM, the signal polarization is reversed to Horizontal Mode (HM) and returns to the PBS common port, being directed to RSOA Aux $\mathrm{D}_{\mathrm{D}}$ with the aid of an HWP. This closes the circulation path of the first cavity.

At the RSOA Aux $x_{D}$, the downstream extinction ratio $\left(E R_{D}\right)$ of the incoming signal is suppressed and the optical carrier is sent back to the FRM by the same previously described optical path. Again, 
the signal polarization is reversed to VM and it is reflected back to RSOA TX $\mathrm{D}_{\mathrm{D}}$, closing the circulation path of the second cavity. The remaining $10 \%$ optical power at the power coupler output is sent to the ONT to be detected and later used for re-modulation. A polarization filter (PF) assures that only the modulated signal is transmitted, reducing unwanted noise.

The wavelength-multiplexed signals are transported over a feed fiber to the Remote Node (RN), where an AWG demultiplexes them, which are then sent to the correspondent ONT by a distribution fiber. At the ONT, $10 \%$ of the incoming signal is diverted by a power splitter to the receiver, for downstream detection. The remaining optical power is sent to the upstream transmitter, where two cascaded-RSOAs erase the downstream data and insert the upstream data into the same carrier.

The carrier-reuse performance is highly dependent on $\mathrm{ER}_{\mathrm{D}}$ and the received optical power, which is related to the link power budget, thus penalizing transmission in the upstream direction. Then, to improve the upstream performance, we enhanced the noise suppression scheme, by adding an extra RSOA at the ONT. This RSOA Aux $x_{U}$ operates in the linear gain mode, to increase optical power of the downstream signal before it reaches the RSOA $\mathrm{Tx}_{\mathrm{D}}$, which, by its turn, operates in deep saturation, to properly erasure the downstream data and then modulate the carrier with upstream data. An optical band pass filter (Op. BPF) reduces the unwanted ASE noise inherent to the RSOA Aux $\mathrm{U}$ and a 4-port optical circulator (Op. Circ.) is responsible for directing the upcoming downstream signal to the RSOA Aux $x_{U}$, next to the RSOA $\mathrm{Tx}_{\mathrm{D}}$ and finally back to the fiber, after being modulated with upstream data.

\section{VALIDATION OF THE SIMULATION FRAMEWORK}

In order to evaluate the proposed topology, we performed numerical simulations using Optisystem 13 , which is a powerful software design tool to plan, test and simulate optical links. In an effort to avoid discrepancies and obtain reliable results, we calibrated our topology accordingly to our earlier experimental investigation [8]. The detailed calibration process and the overall numerical evaluation for the double-cavity self-seeding topology with carrier reuse can be found in [29].

The main concerns on these calibrations were to reproduce the RSOAs characteristics and the Bit Error Rate (BER) performances on both downstream and upstream direction. We initially performed a numerical calibration of the RSOAs gain curves to match the gain profile presented by the actual RSOAs used in our experimental investigation. Fig. 3(a) shows the comparison between the gains curves for the actual RSOA and the Optisystem model under 60 and $90 \mathrm{~mA}$ biases current. As it can be seen, the values differ less than, respectively, $0.3 \mathrm{~dB}$ and $1.0 \mathrm{~dB}$ from the experimental data. Therefore, we have satisfactory reproduced the optical behavior of the devices operating on $\mathrm{CW}$ mode. We have also confirmed that the simulated optical spectrum profile after is very close to the one obtained in the experiments, reinforcing that the RSOA is well calibrated (see Fig. 3(b)).

As shown in Fig. 3(c), the BER curves for a downstream extinction ratio of 4 and $5 \mathrm{~dB}$ were also validated in the simulation setup by adjusting the photodetector parameters. For this downstream 
transmission, a $20 \mathrm{~km}$ optical link was considered and the self-seeding output power in the first roundtrip was set to $-27 \mathrm{dBm}$. We have observed a maximum error of $0.4 \mathrm{~dB}$ for the curve with $\mathrm{ER}_{\mathrm{D}}=5.0$ $\mathrm{dB}$ and $0.2 \mathrm{~dB}$ for the curve with $\mathrm{ER}_{\mathrm{D}}=4.0 \mathrm{~dB}$.

For the upstream calibration, we have first fit the BER performance for a RSOA $\mathrm{TX}_{U}$ optical injection power of $-12 \mathrm{dBm}$ and $\mathrm{ER}_{\mathrm{D}}=0 \mathrm{~dB}$, in order to simulate the best optical erasure condition. Then, we kept the same set of calibration parameters to generate the BER curve for $\mathrm{P}_{\text {inj }}=-17 \mathrm{dBm}$ with $\mathrm{ER}_{\mathrm{D}}=3.5 \mathrm{~dB}$. These comparisons are shown in Fig. 3(d), where an excellent agreement can be observed. Hence, we consider that the simulation setup was calibrated with our experimental test bed on both directions and further extrapolations are expected to be reliable.
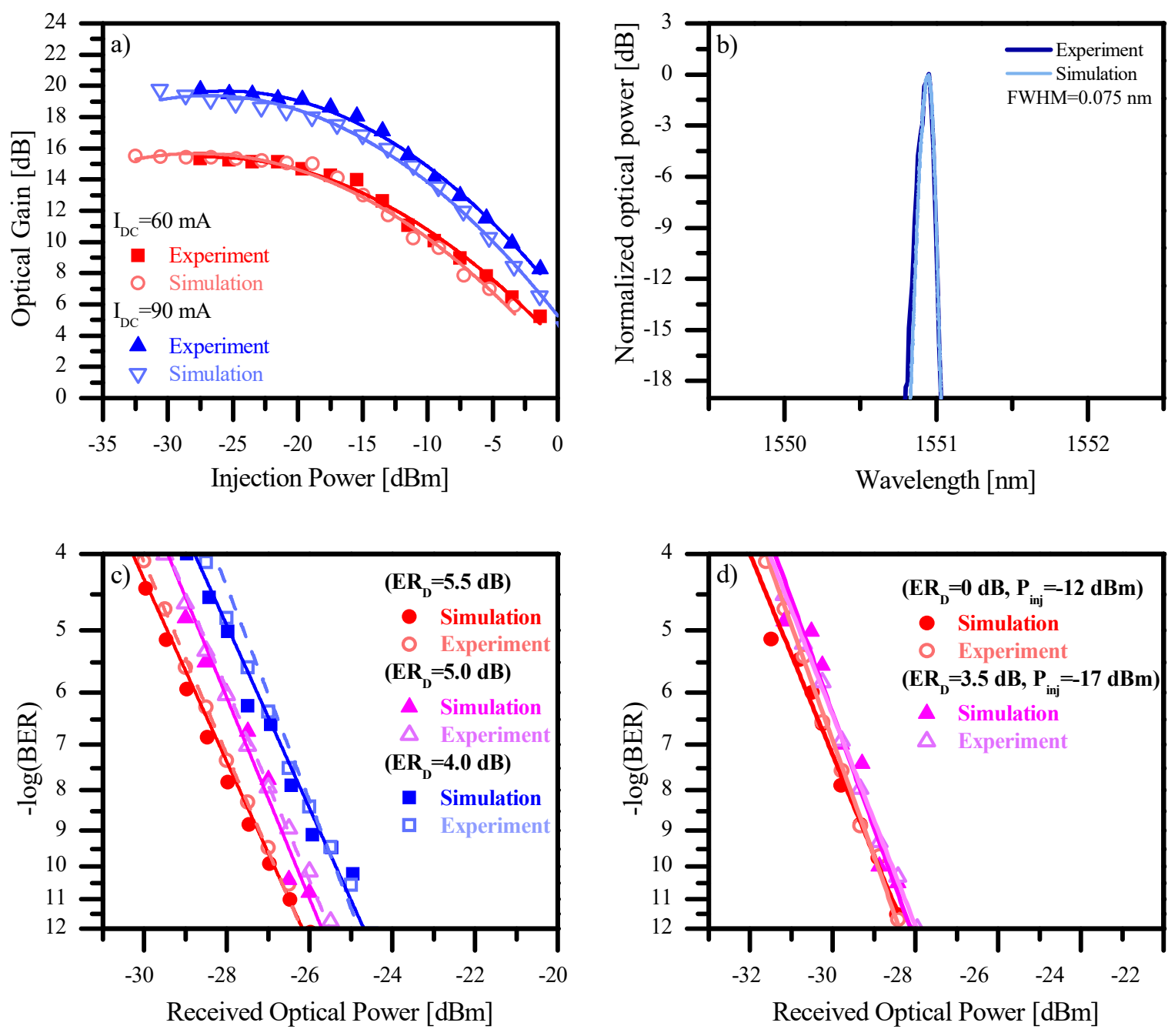

Fig. 3. Comparisons between simulated and experimental data in the calibration process. a) RSOAs gain profile. b) Selfseeding output signal spectrum after the polarization filter. c) Downstream BER performance. d) Upstream BER performance.

\section{Simulation SETUP}

The simulation setup for downstream transmission is depicted in Fig. 4. The RSOA Tx $x_{D}$ is biased at $100 \mathrm{~mA}$ as well as directly modulated by a RF signal. The RSOA Aux $\mathrm{D}_{\mathrm{D}}$ is biased by a $125 \mathrm{~mA} \mathrm{DC}$ current, in order to provide enhanced data suppression. The RF signal is composed by a 
pseudorandom bit sequence $\left(\mathrm{k}=2^{9}-1\right)$ modulated to the carrier by using a digital modulation format. We have run tests at data rates of 155,622 and $1250 \mathrm{Mbps}$, over carriers of $1.25,2.5$ and $5 \mathrm{GHz}$ employing ASK, BPSK, QPSK, 8PSK and 16 QAM formats. The modulated output optical signal passes through a PBS with polarization rejection of $25 \mathrm{~dB}$ and the wavelength is selected by the AWG. For the simulation, the link wavelength is set at $1550.91 \mathrm{~nm}$. The AWG is composed of optical filters of $4^{\text {th }}$. order, $0.5 \mathrm{~nm}$ bandwidth and insertion loss of $5.5 \mathrm{~dB}$. After wavelength selection, $90 \%$ of the signal power goes to the FRM and $10 \%$ is effectively transmitted to the fiber, using a power coupler and a PF. The FRM has a reflectance of $99 \%$ and insertion loss of $0.5 \mathrm{~dB}$. In the simulations, in order to reach dynamical stability, the complete round-trip path in the cavity is performed 150 times before any data insertion. The data circulation process takes another 20 round-trips and the last 3 are used for performance analysis.

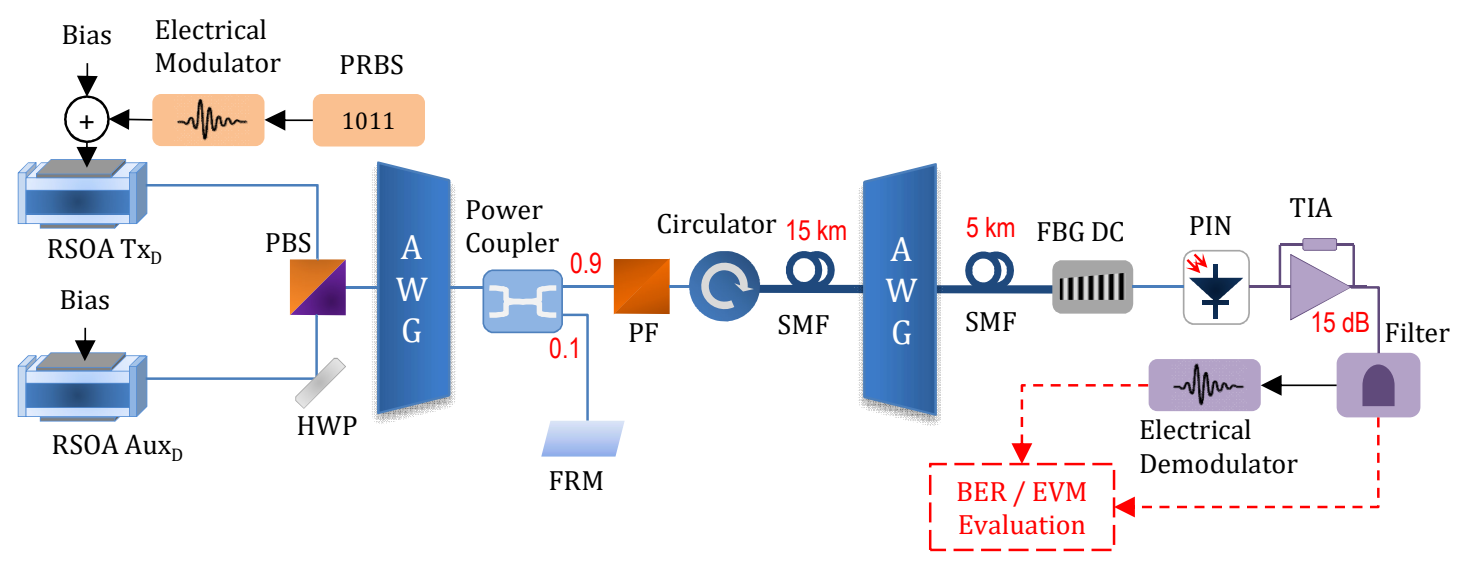

Fig. 4. Simulation setup for downstream transmission. The BER/EVM evaluation is performed before the RF transmitter processing, which is set right after the TIA. We evaluated carriers at 1.25, 2.5 and $5 \mathrm{GHz}$ transmitting 155, 622 and 1250 Mbps using ASK and BPSK, QPSK, 8PSK and 16QAM modulation formats.

The transmitted signal passes $15 \mathrm{~km}$ of SMF-25 fiber, the feeding fiber. At the remote node, another AWG with the identical characteristics selects the desired channel for another $5 \mathrm{~km}$ of fiber transport, the drop fiber. At the receiver, we have noticed the already mentioned power fading problem due to chromatic dispersion. This is because the sidebands are affected differently by chromatic dispersion (CD) and suffer distinct phase delays. As a result, their beating on the PD causes periodic dips on the RF received power (Fig. 5). To address this issue, we employed a Fiber-BraggGrating (FBG) for dispersion compensation, with enough bandwidth for a single channel and an insertion loss of only $3.5 \mathrm{~dB}$, providing $335 \mathrm{ps} / \mathrm{nm}$ of CD compensation.

The PIN photodiode was calibrated to 0.5 responsivity, $10^{-22} \mathrm{~W} / \mathrm{Hz}$ thermal noise power density, 10 $\mathrm{nA}$ of dark current and a Gaussian shot noise distribution. These values were chosen to emulate the overall noise of the optical receiver, including a Transimpedance Amplifier (TIA), which provides a gain of $15 \mathrm{~dB}$, and the remaining experimental elements. At this point, we evaluated the BER and Error Vector Magnitude (EVM) characteristics of the RF signal to be delivered to the wireless transmitter. Both evaluations are provided by the software tools. 


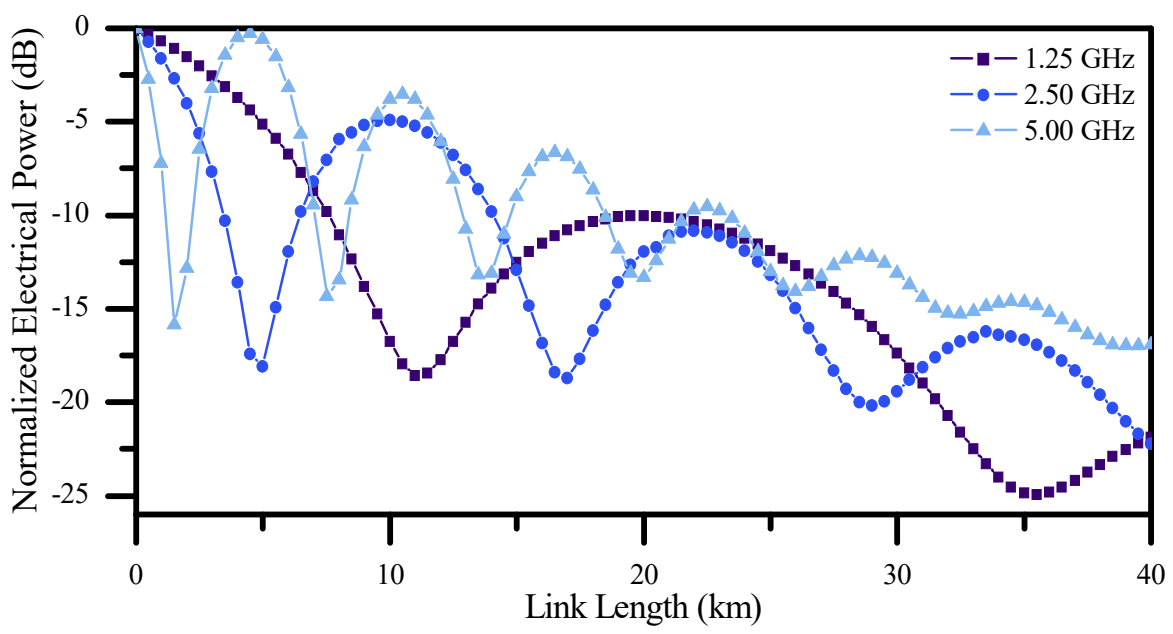

Fig. 5. Fading nodes due to fiber chromatic dispersion for 1.25, 2.5 and $5 \mathrm{GHz}$ ASK transmission.

The simulation setup for upstream transmission is depicted at Fig. 6. In this case, the downstream signal is extracted right after the FBG (see Fig. 4) and $90 \%$ of its total power is used as an input for the transmission setup. The bidirectional power coupler ratio is set to $60 / 40$ in order to guarantee that at least $60 \%$ of the downstream power is directed to the RSOA Aux $\mathrm{X}_{U}$ through the 4-port circulator and, as a consequence, $40 \%$ of the upstream power emerging the $\mathrm{RSOA} \mathrm{Tx}_{\mathrm{U}}$ is sent back to the distribution fiber in the reverse direction. This power coupler ratio was is initially set to 50/50 and then optimized to $60 / 40$ in order to provide a better BER performance.

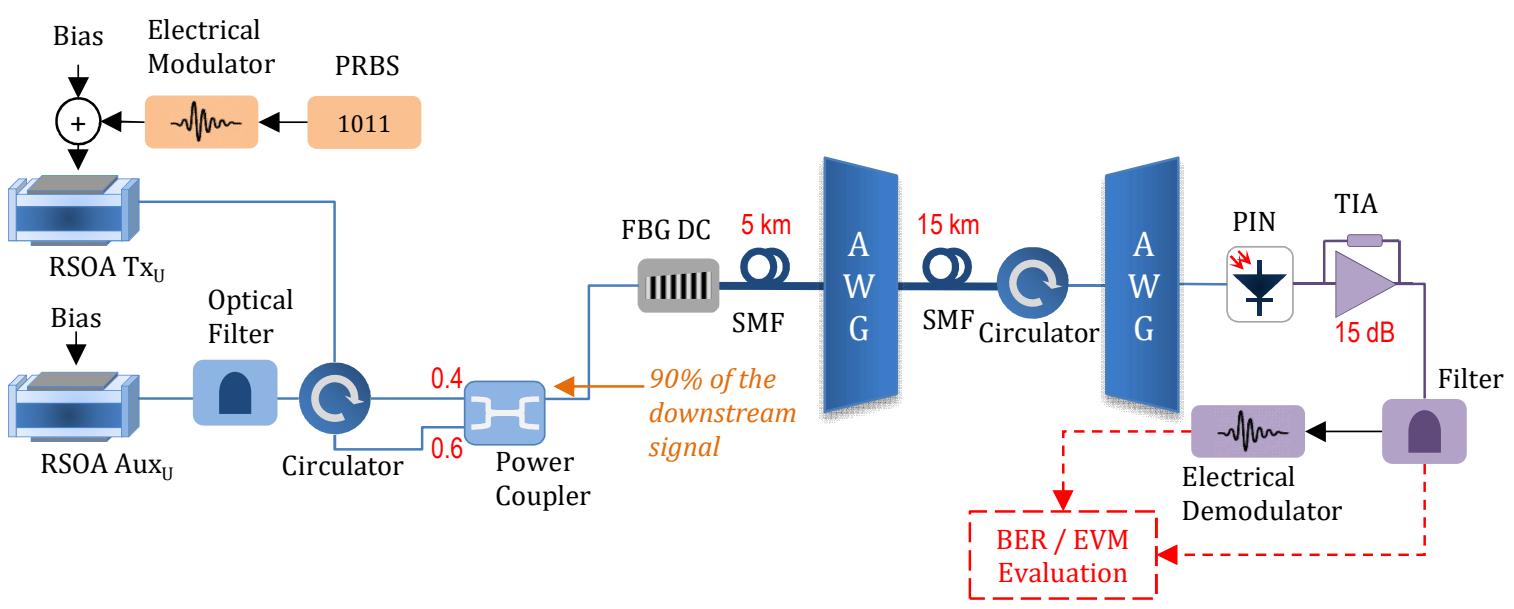

Fig. 6. Simulation setup for upstream transmission. We evaluated carriers at $1.25,2.5$ and $5 \mathrm{GHz}$ transmitting 155,622 and 1250 Mbps using ASK and BPSK, QPSK, 8PSK and 16QAM modulation formats, exactly as it was done for downstream.

At the ONT, the RSOA Aux is biased by a $125 \mathrm{~mA} \mathrm{DC}$ current and works as an amplifier to the incoming downstream signal, to guarantee a $-6 \mathrm{dBm}$ injected optical power to the RSOA Tx $\mathrm{U}$. At this level of injected power, RSOA Tx $\mathrm{x}_{\mathrm{U}}$ works on saturation regime (biased at $100 \mathrm{~mA}$ ) and it is able to erase the downstream data, to subsequently modulate the upstream data. A band-pass optical filter with a $0.65 \mathrm{~nm}$ bandwidth is used to mitigate the ASE noise added by the RSOA Aux . The remaining elements are the same as described for the downstream topology. The RF signals chosen to 
evaluate the upstream performance are also the same as the downstream case. The downstream and upstream links are evaluated separately.

\section{RESULTS AND DISCUSSION}

We analyzed the downstream BER for carriers at 1.25, 2.5 and $5 \mathrm{GHz}$ transmitting 155, 622 and 1,250 Mbps using the ASK modulation format, as shown in Figs. 7(a) to 7(c). All transmitted signals allowed successful recovery with an optical received power of $-25 \mathrm{dBm}$ when considering a BER Forward Error Correction (FEC) limit of $10^{-3}$. The $5 \mathrm{GHz}$ signal reaches a $10^{-9} \mathrm{BER}$ floor even for 155 Mbps transmission. This is because both RSOAs being considered presented a typical $3 \mathrm{GHz} \mathrm{E} / \mathrm{O}$ bandwidth [30], introducing losses of 1.5 and $5.5 \mathrm{~dB}$ for the respective frequency bands, as well as due to the decrease in the optical power level circulating within the cavity at this modulation frequency. The performance also moderately worsens with increasing bit rate, reaching a $10^{-8} \mathrm{BER}$ floor when transmitting $1.25 \mathrm{Gbps}$.
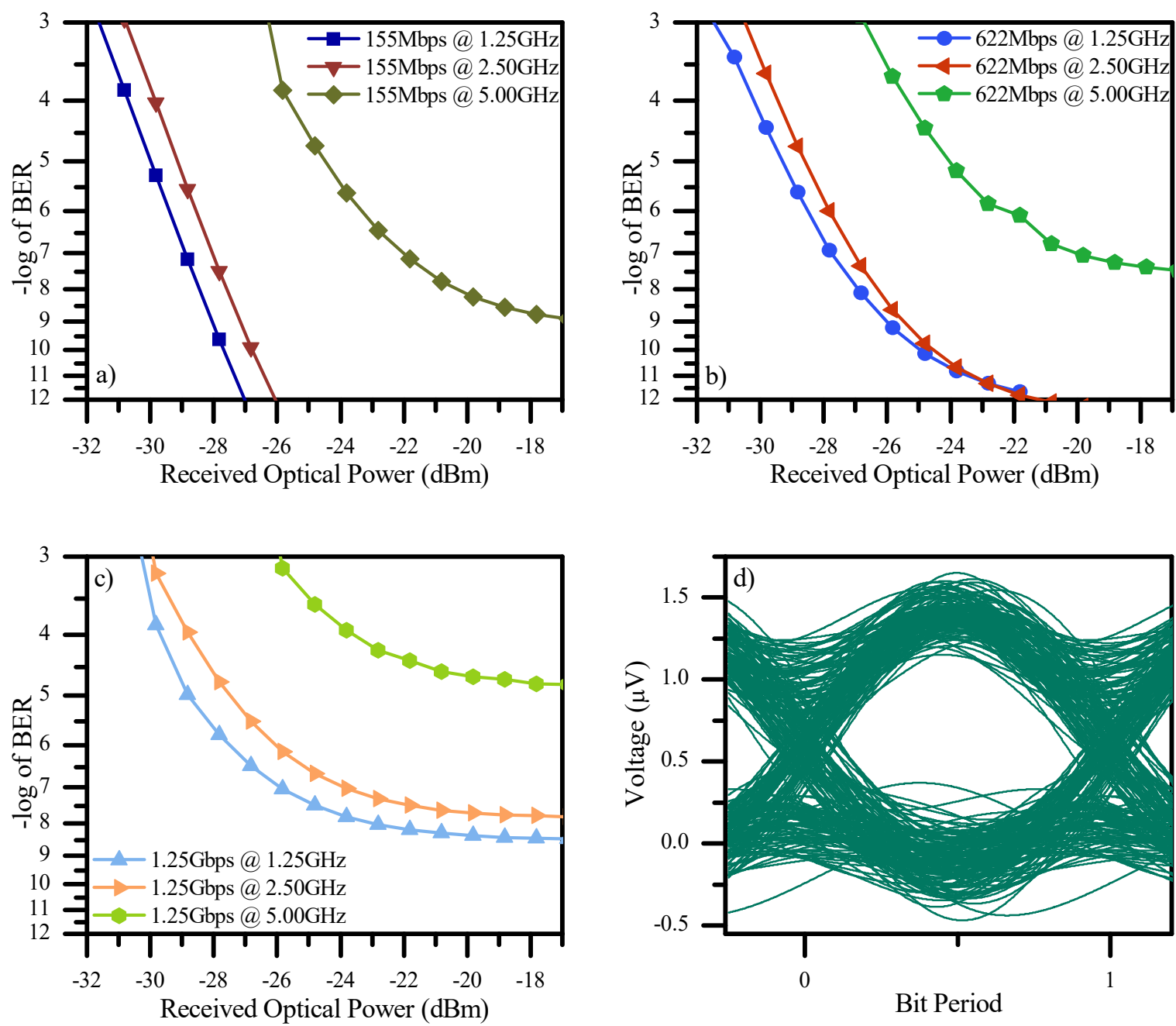

Fig. 7. Downstream BER curves for carriers at 1.25, 2.5 and $5 \mathrm{GHz}$ transmitting a) $155 \mathrm{Mbps}$, b) $622 \mathrm{Mbps}$ and c) $1.25 \mathrm{Gbps}$ using the ASK modulation format. d) Eye diagram for a $1.25 \mathrm{GHz}$ carrier with $1.25 \mathrm{Gbps}$ and optical received power of $26.82 \mathrm{dBm}$. 
A typical eye diagram resulting from the downstream transmission is provided in Fig. 7(d). This result was obtained from a $1.25 \mathrm{Gbps}$ ASK transmission at $1.25 \mathrm{GHz}$ with a received optical power of $-26.84 \mathrm{dBm}$. The eye vertical aperture indicates the moderate presence of noise and the horizontal aperture also indicates moderate jitter. The main sources of noise are the residual noise introduced in the self-seeding cavity due to incomplete data erasure and the noise added by the optical receiver. It is worth noticing we allowed a residual dispersion on the link, in order to emulate imprecise dispersion compensation. In addition, the linewidth of the self-seeding carrier is very large when compared to conventional single-mode lasers ( $\Delta \lambda=0.095 \mathrm{~nm}$ at $1550.91 \mathrm{~nm}$ ), also contributing for the degradation of optical signals. However, since the eye pattern appears wide open on both dimensions, we assume that the self-seeding process produces a fair signal quality for our applications.

For the IQ modulation formats, we investigated the transmission of BPSK, QPSK, 8PSK and 16QAM signals at $1.25 \mathrm{GHz}$ with a bit rate of $1.25 \mathrm{Gbps}$. The EVM evaluations (Fig. 8) were all performed at a received optical power of $-26.84 \mathrm{dBm}$, which is the measured value at reception without any amplification. Higher order formats were not tested due to computational limitations.
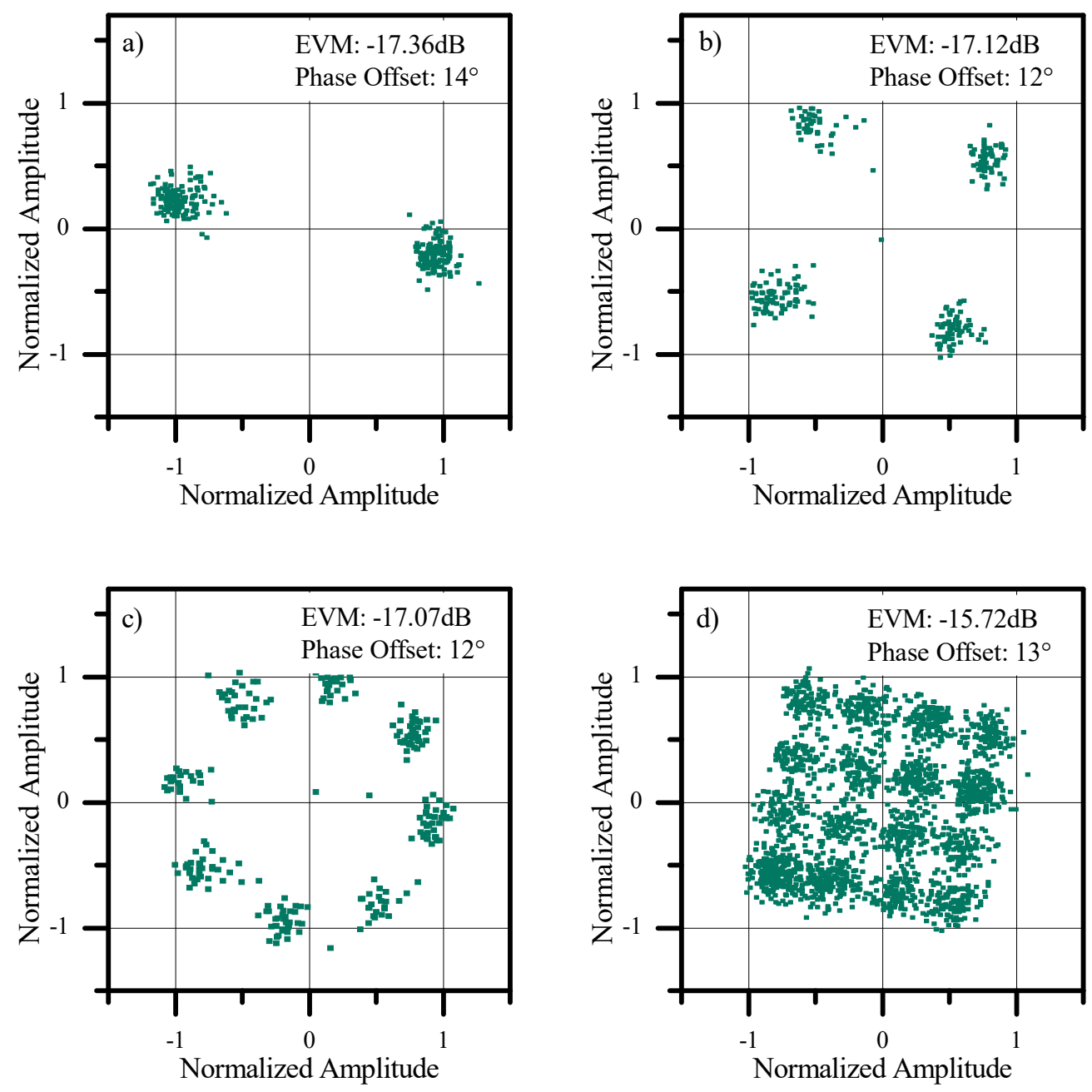

Fig. 8. Downstream constellations for a) BPSK, b) QPSK, c) 8PSK and d) 16QAM signals at $1.25 \mathrm{GHz}$ with a bit rate of $1.25 \mathrm{Gbps}$. The EVM evaluations were all performed at a received optical power of $-26.84 \mathrm{dBm}$. 
Taking the LTE Technical specification provided by $3 \mathrm{GPP}$ as a reference [31], the minimum required EVM for BPSK and QPSK is $-15.31 \mathrm{~dB}(17.5 \%)$ and for 16QAM is $-18.06 \mathrm{~dB}(12.5 \%)$. There is no indication for 8PSK, but the requirement must be between these previous values. Therefore, our results are close to the minimum requirements, with only 16QAM falling short. The constellations also present a phase offset of little more than 10 degrees on all signals. We believe that this phase rotation is imposed by the transfer functions of the optical filters that emulate the AWGs both on transmission and reception. However, since this deviation is expected to be constant, this can be easily handled with simple signal processing techniques. We chose not to handle these deviations in the software to avoid interfering with EVM values and the performance analysis.

We also analyzed the upstream BER for carriers at 1.25, 2.5 and $5 \mathrm{GHz}$ transmitting 155, 622 and 1,250 Mbps using the ASK modulation format, as shown in Figs. 9(a) to 9(c). All transmitted signals were successfully detected with an optical received power of $-28 \mathrm{dBm}$. It is important to mention $\mathrm{ER}_{\mathrm{U}}$ is higher than $\mathrm{ER}_{\mathrm{D}}$ since it is not limited by an erasure efficiency requirement; therefore, downstream and upstream performances are not directly comparable.
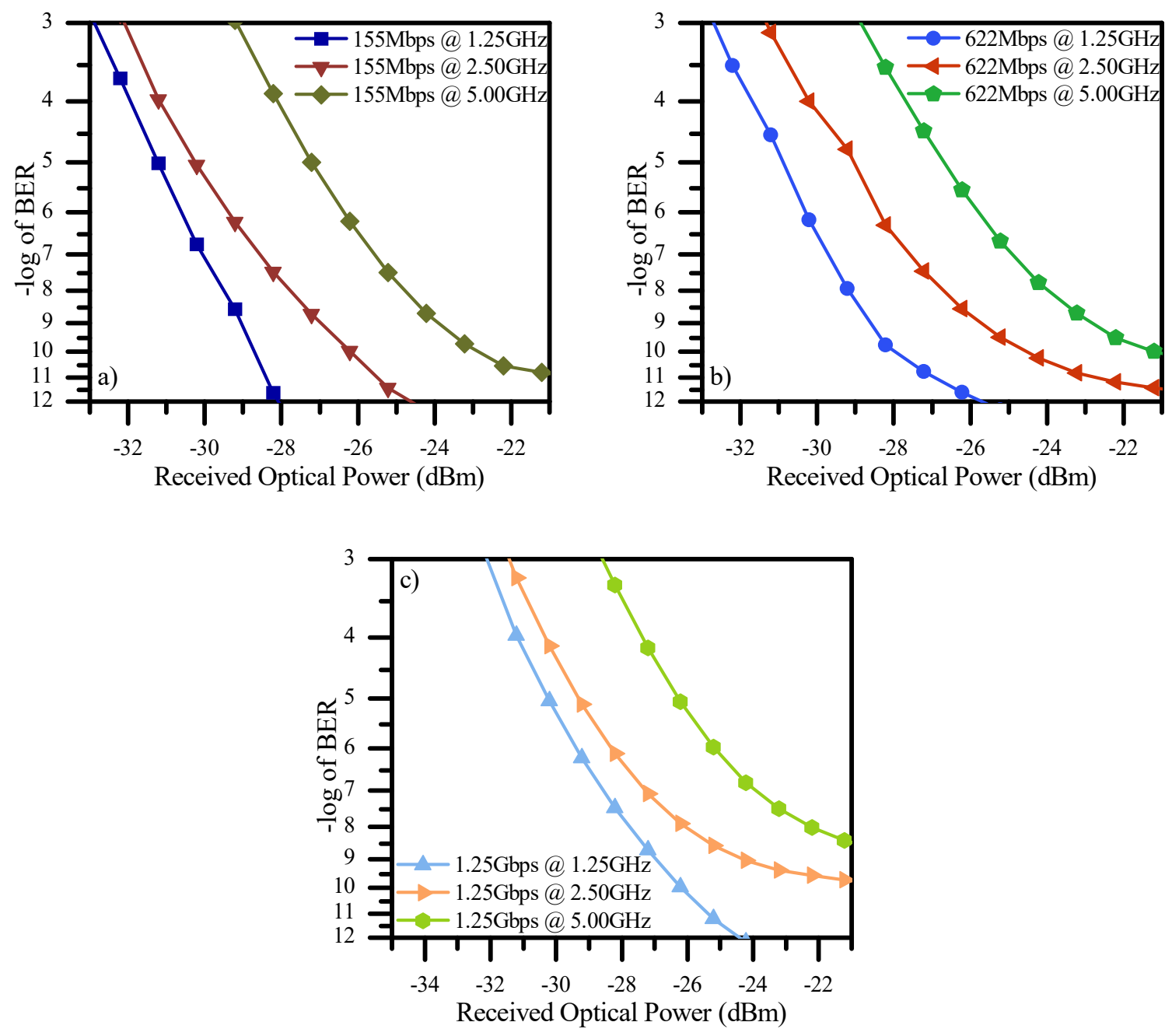

Fig. 9. Upstream BER curves for carriers at 1.25, 2.5 and $5 \mathrm{GHz}$ transmitting a) $155 \mathrm{Mbps}$, b) $622 \mathrm{Mbps}$ and c) $1.25 \mathrm{Gbps}$ using the ASK modulation format. 
Regarding the IQ modulation formats, we also evaluated the EVM of BPSK, QPSK, 8PSK and 16QAM signals at $1.25 \mathrm{GHz}$ with a bit rate of $1.25 \mathrm{Gbps}$ and a received optical power of $-26.84 \mathrm{dBm}$. The electrical constellations and EVM values are displayed in Fig. 10. In this case, the transmission performance is slightly worse that the downstream counterpart, although they are also not directly comparable. Still, the results met the minimum requirements by the same LTE specification criteria that were used before, with the exception of 16QAM.

Finally, although the results presented here are satisfactory, it is worth noticing that we have concentrated our efforts on calibrating the simulation setups as close as possible to the experimental investigations in order to achieve reliability. Therefore, the components and operation conditions were not optimized for the transmission of RF signals and the aimed application and these results can be improved even further. As a future work, we intend to optimize these simulation frameworks and experimentally investigate the fronthaul topologies presented here with new $5 \mathrm{G}$ radio modulation formats.
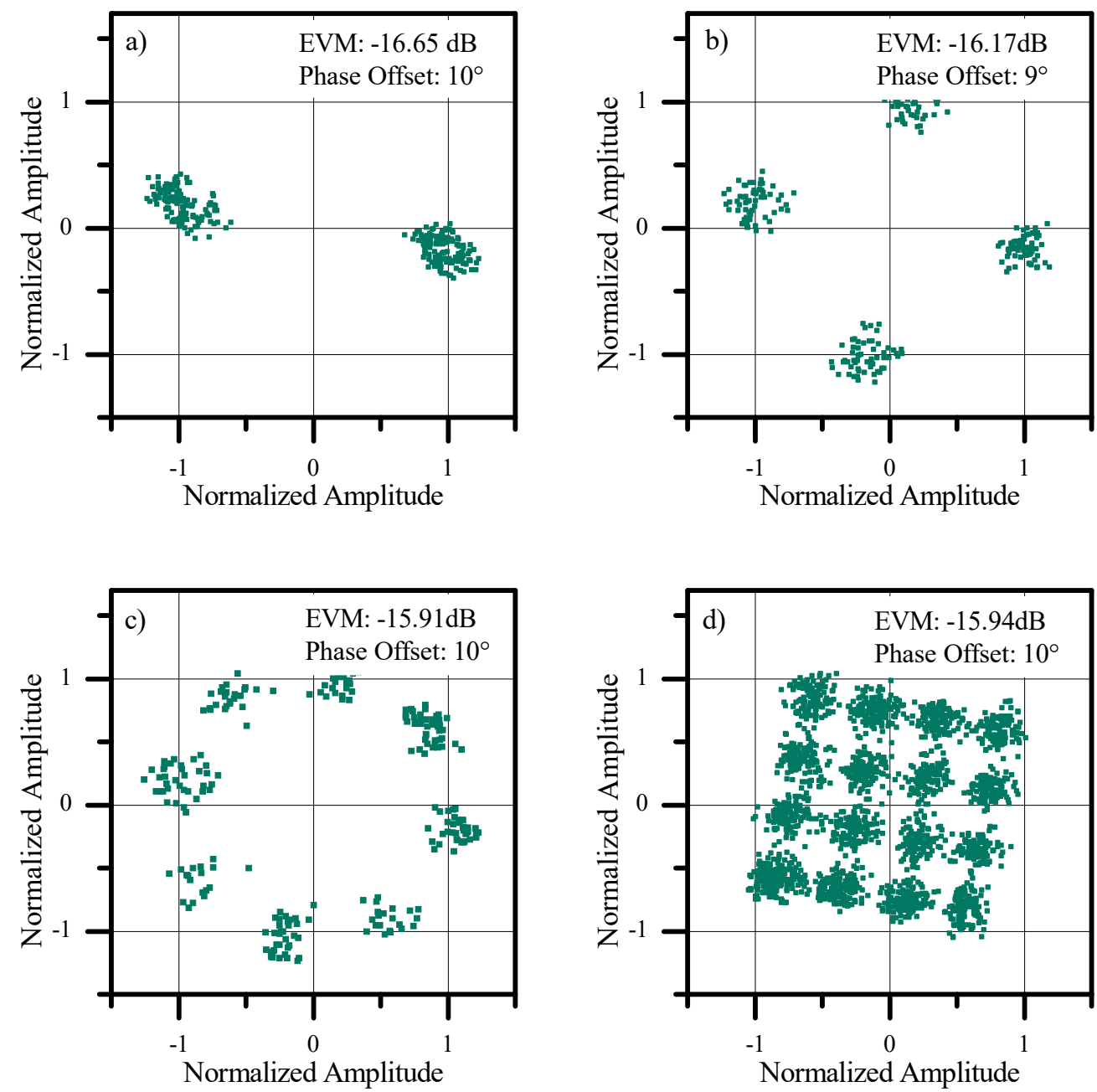

Fig. 10. Upstream constellations for a) BPSK, b) QPSK, c) 8PSK and d) 16QAM signals at $1.25 \mathrm{GHz}$ with a bit rate of 1.25 Gbps. The EVM evaluations were all performed at a received optical power of $-26.84 \mathrm{dBm}$. 


\section{CONCLUSION}

In this paper, we proposed and numerically evaluated a combined double cavity self-seeded and cascaded-RSOAs carrier-reuse colorless WDM-PON network serving as an analog fronthaul. The computational setup was validated against experimental results and BER calculations show a $20 \mathrm{~km}$ bidirectional successful transmission of ASK signals in the wireless bands of $1.25,2.5$ and $5 \mathrm{GHz}$ with data rates of 155, 622 and 1250 Mbps. We also evaluated that the EVM performance of BPSK, QPSK, 8PSK and 16QAM signals at $1.25 \mathrm{GHz}$ with a $1.25 \mathrm{Gbps}$ bit rate is close to the required limits imposed by LTE standard, demonstrating the proposal feasibility for future mobile fronthauls.

\section{ACKNOWLEDGMENTS}

The authors would like to thank the financial support provided by CAPES and CNPq.

\section{REFERENCES}

[1] M. A. Romero, "Passive Optical Networks: Present Status and Future Outlook", in 2017 SBMO/IEEE MTT-S International Microwave and Optoelectronics Conference (IMOC), 2017.

[2] C. Wagner, M. H. Eiselt, M. Lawin, S. J. Zou, K. Grobe, J. J. V. Olmos, and I. T. Monroy, "Impairment Analysis of WDM-PON Based on Low-Cost Tunable Lasers", J. Light. Technol., vol. 34, no 22, p. 5300-5307, 2016.

[3] A. Maho, G. Simon, S. Barbet, F. Lelarge, F. Saliou, P. Chanclou, P. Parolari, L. Marazzi, M. Brunero, M. Martinelli, S. A. Grebewold, J. Leuthold, and R. Brenot, "Demystification of the Self-Seeded WDM Access", $J$. Light. Technol., vol. 34, n 2, p. 776-782, 2016.

[4] E. Wong, K. L. Lee, and T. B. Anderson, "Directly Modulated Self-Seeding Reflective Semiconductor Optical Amplifiers as Colorless Transmitters in Wavelength Division Multiplexed Passive Optical Networks", J. Light. Technol., vol. 25, $\mathrm{n}^{\circ}$ 1, p. 67-74, 2007.

[5] M. Presi, A. Chiuchiarelli, R. Corsini, and E. Ciaramella, "Self-seeding of semiconductor lasers for next-generation WDM passive optical networks", Int. Conf. Transparent Opt. Networks, p. 3-6, 2013.

[6] S. A. Gebrewold, L. Marazzi, P. Parolari, R. Brenot, S. P. Ó. Duíll, R. Bonjour, D. Hillerkuss, C. Hafner, and J. Leuthold, "Reflective-SOA fiber cavity laser as directly modulated WDM-PON colorless transmitter", IEEE J. Sel. Top. Quantum Electron., vol. 20, $\mathrm{n}^{\circ}$ 5, 2014.

[7] P. Parolari, M. Brunero, R. Brenot, and M. Martinelli, "Up to 20-Gb/s multilevel WDM PON transmitter based on self-seeded RSOAs", in 2015 European Conference on Optical Communication (ECOC), 2015.

[8] U. R. Duarte, R. S. Penze, F. R. Pereira, F. F. Padela, J. B. Rosolem, and M. A. Romero, "Combined Self-Seeding and Carrier Remodulation Scheme for WDM-PON", J. Light. Technol., vol. 31, nº 8, p. 1323-1330, 2013.

[9] D. R. Celino, U. R. Duarte, and M. A. Romero, "Improved Self-Seeding and Carrier Remodulation Performance for WDM-PON by Means of Double RSOA Erasure", submitted for publication J. Opt. Commun. Netw.

[10] D. Nesset, "PON Roadmap [Invited]", IEEE/OSA J. Opt. Commun. Netw., vol. 9, no 1, p. 71-76, 2017.

[11] A. Pizzinat, P. Chanclou, F. Saliou, and T. Diallo, "Things You Should Know About Fronthaul”, J. Light. Technol., vol. 33, n 5, p. 1077-1083, 2015.

[12] "Common Public Radio Interface (CPRI), Interface Specification, v5.0", 2011. [Online]. Available at: http://www.cpri.info. [Sept 27, 2018].

[13] "Open Base Station Architecture Initiative ", 2002. [Online]. Available at: http://www.obsai.com. [Sept 27, 2018].

[14] G. K. Chang and L. Cheng, "The benefits of convergence", Phil. Trans. R. Soc. A, vol. 374, no 2062, 2016.

[15] F. Saliou, G. Simon, P. Chanclou, M. Brunero, L. Marazzi, P. Parolari, and M. Martinelli, "Self - Seeded RSOAs WDM PON Field Trial for Business and Mobile Fronthaul Applications", Conf. Opt. Fiber Commun. Tech. Dig. Ser., vol. 2015-June, no 1, p. 7-9, 2015.

[16] D. Thierno, A. Pizzinati, F. Saliou, P. Chanclou, G. Simon, and C. Aupetit-Berthelemot, "Self-Seeded DWDM Solution for Fronthaul Links in Centralized-Radio Access Network", J. Light. Technol., vol. 34, no 21, p. 49654971, 2016.

[17] Y. Ma, Z. Xu, C. Zhang, H. Lin, Q. Wang, M. Zhou, H. Wang, J. Yu, and X. Wang, "Demonstration of digital fronthaul over self-seeded WDM-PON in commercial LTE environment", Opt. Express, vol. 23, n 9, p. 11927, 2015.

[18] A. M. Souza, D. R. Celino, U. R. Duarte, and M. A. Romero, "Analog Radio-over-Fiber Fronthaul by a Double Cavity Self-Seeded WDM-PON", in Simpósio Brasileiro de Microondas e Optoeletrônica - MOMAG, 2018.

[19] T. Pfeiffer, "Next Generation Mobile Fronthaul Architectures", in Optical Fiber Communication Conference, 2015.

[20] X. Liu and F. Effenberger, "Emerging Optical Access Network Technologies for 5G Wireless", J. Opt. Commun. Netw., vol. 8, no 12, p. B70, 2016.

[21] A. Checko, H. L. Christiansen, Y. Yan, L. Scolari, G. Kardaras, M. S. Berger, and L. Dittmann, "Cloud RAN for Mobile Networks-A Technology Overview”, IEEE Commun. Surv. Tutorials, vol. 17, nº 1, p. 405-426, 2015.

[22] L. Cheng, X. Liu, N. Chard, F. Effenberger, and G. Chang, "Experimental Demonstration of Sub-Nyquist Sampling 
for Bandwidth- and Hardware-Efficient Mobile Fronthaul Supporting $128 \times 128$ MIMO with 100-MHz OFDM Signals", Opt. Fiber Commun. Conf. 2016, 2016.

[23] M. Xu, F. Lu, J. Wang, L. Cheng, D. Guidotti, and G.-K. Chang, "Key Technologies for Next-Generation Digital RoF Mobile Fronthaul With Statistical Data Compression and Multiband Modulation", J. Light. Technol., vol. 35, no 17 , p. 3671-3679, 2017.

[24] C. Ranaweera, E. Wong, A. Nirmalathas, C. Jayasundara, and C. Lim, "5G C-RAN With Optical Fronthaul: An Analysis From a Deployment Perspective", J. Light. Technol., vol. 36, nº 11, p. 2059-2068, 2018.

[25] U. Gliese, S. Norskov, and T. N. Nielsen, "Chromatic dispersion in fiber-optic microwave and millimeter-wave links", IEEE Trans. Microw. Theory Tech., vol. 44, n 10, p. 1716-1724, 1996.

[26] J. Ma, J. Yu, C. Yu, X. Xin, J. Zeng, and L. Chen, "Fiber Dispersion Influence on Transmission of the Optical Millimeter-Waves Generated Using LN-MZM Intensity Modulation”, J. Light. Technol., vol. 25, n 11, p. 32443256, 2007.

[27] S. Liu, M. Xu, J. Wang, F. Lu, W. Zhang, H. Tian, and G.-K. Chang, "A Multilevel Artificial Neural Network Nonlinear Equalizer for Millimeter-Wave Mobile Fronthaul Systems”, J. Light. Technol., vol. 35, no 20, p. 44064417, 2017

[28] J. Wang, "Nonlinear Impairments and Mitigation Technologies for the Next Generation Fiber-Wireless Mobile." Ph.D. thesis, Georgia Institute of Technology, Atlanta, 2017.

[29] D. R. Celino, "Proposta e Análise de redes WDM-PON Empregando Auto- Alimentação e Dupla Cavidade Óptica São Carlos." M.Sc. thesis, University of Sao Paulo, Sao Carlos, 2017.

[30] P. Parolari, L. Marazzi, M. Brunero, M. Martinelli, R. Brenot, A. Maho, S. Barbet, G. Gavioli, G. Simon, S. D. Le, F. Saliou, and P. Chanclou, "C- and O-Band Operation of RSOA WDM PON Self-Seeded Transmitters up to $10 \mathrm{~Gb}$ / s [ Invited ]”, J. Opt. Commun. Networks, vol. 7, nº 2, p. 249-255, 2015.

[31] LTE: Evolved Universal Terrestrial Radio Access (E-UTRA) - User Equipment (UE) radio transmission and reception (3GPP TS 36.101 version 10.3.0 Release 10), 2011. 